\title{
The effect of initial molecular profile on response to recombinant interferon alpha (rIFNa) treatment in early myelofibrosis
}

Richard T. Silver, MD ${ }^{a}$, Ariella Barel ${ }^{a}$, Elena Lascu ${ }^{a}$, Ellen K. Ritchie, MD ${ }^{a}$, Gail J. Roboz, MD ${ }^{a}$, Paul Christos ${ }^{b}$, Attilio Orazi, MD ${ }^{c}$, Duane Hassane, PhD $^{d}$, Wayne Tam, MD, $\mathrm{PhD}^{\mathrm{c}}$ and Nicholas C. P. Cross, PhD
a. Richard T Silver Myeloproliferative Neoplasm Center, Division of Hematology-Medical Oncology, Weill Cornell Medicine, New York, NY USA
b. Division of Biostatistics and Epidemiology, Department of Healthcare Policy \& Research, Weill Cornell Medicine, New York, NY, USA
c. Department of Pathology and Laboratory Medicine, Weill Cornell Medicine, New York, NY, USA
d. Institute for Computational Biomedicine, Division of Hematology and Medical Oncology, Department of Medicine, Weill Cornell Medicine, New York, NY, USA
e. Faculty of Medicine, University of Southampton, Southampton, UK

Corresponding Author: Richard T. Silver, MD

Mailing Address:

Weill Cornell Medicine

1305 York Ave, Box \# 403

New York, NY 10021

Phone \#: 646-962-2255

Fax \#: 646-962-1607

Email: rtsilve@med.cornell.edu

Running title: rIFNa treatment in early MF

Number of text pages: 20

Number of Tables: 4

Number of Figures: 1

\section{Funding:}

This work was supported by the William and Judy Higgins Trust and the Johns Family Fund of the Cancer Research and Treatment Fund Inc., New York, NY, USA.

Dr. Paul Christos was partially supported by a grant from the Clinical and Translational Science Center at Weill Cornell Medicine (UL1-TR000457-06). DCH is supported by the Leukemia and Lymphoma Society (LLS TRP\#R. to DCH).

\section{Disclosures:}

Richard T. Silver, MD: Consultancy, PharmaEssentia, AOP Orphan, Incyte, Gilead Sciences 
Ellen K. Ritchie, MD: Honorarium and Research Funding, Novartis \& Pfizer; Speaker Bureau, Celgene, Incyte, Ariad

Gail J. Roboz, MD: Consultancy, Agios, Amgen, Amphivena, Astex, AstraZeneca, Boehringer Ingelheim, Celator, Celgene, CTI Biopharma, Genoptix, Janssen, Junno, Medimmune, MEI Pharma, Novartis, Onconova, Pfizer, Roche, Sunesis \& Teva

Attilio Orazi, MD: Honoraria, Novartis

Nicholas C.P. Cross, PhD.: Consultancy, Honoraria, Research Funding and Speaker Bureau, Novartis

Ariella Barel, Elena Lascu, Paul Christos, Duane Hassane, PhD and Wayne Tam, MD, $\mathrm{PhD}$ declare no competing financial interests.

No pharmaceutical support for interferon was offered or requested for this study.

\section{Contribution:}

RTS designed the study, examined patients, reviewed data and marrow biopsies, wrote the paper.

$A B, E L$ collected and analyzed data, wrote the paper.

EKR, GJR examined patients, reviewed data.

PC performed statistical analyses, wrote the paper.

AO reviewed marrow biopsies, reviewed data.

DH, WT, NCPC performed molecular analyses, reviewed data, wrote the paper.

\section{Precis:}

1) Early treatment of myelofibrosis with low-dose interferon led to stabilization or improvement in $73 \%$ of patients with acceptable toxicity.

2) Neither initial driver mutation status nor risk score correlated with response to therapy, but patients with ASXL1 and SRSF2 did poorly.

\section{Prior presentations:}

Presented in abstract form at the 58 annual meeting of the American Society of Hematology, San Diego, California, December 5, 2016.

Presented in abstract form at the XI International CML \& MPN Post-ASH Workshop, Estancia, La Jolla, California, December 6, 2016. 


\section{Abstract:}

Background:

Although recombinant interferon-alpha (rIFNa) effectively treats early myelofibrosis (eMF) patients (pts), the effect of driver and high molecular risk (HMR) mutations (muts) has not been considered. In this Phase II study, we correlate for the first time response to rIFNa treatment with driver and HMR muts.

\section{Methods:}

Pts were diagnosed using WHO or IWG-MRT criteria. Only pts with low or intermediate 1 DIPSS score with $\geq 15 \%$ hematopoietic marrow foci were included. History, symptom assessment, physical exam, blood and marrow studies were performed. Genomic DNA was extracted from frozen cells; next generation targeted sequencing of 45 genes was performed. Either rIFNa-2b, 0.5 mu TIW or PEG-rIFNa-2a, 45 mcg weekly with escalation was initiated. All pts were followed at our institution; regular marrow biopsies were encouraged. IWG-MRT and ELN treatment response criteria was used.

Results:

Of 30 pts (16 women, 14 men; median 58 yrs), 22 were low risk and 8 intermediate 1. Two pts achieved complete remission, 9 partial, 4 clinical improvement, 7 stable disease; 3 progressed, 1 relapsed and 4 died. There were 22 JAK mutated pts, 6 CALR, and 2 MPL. $73 \%$ of pts improved or remained stable with acceptable toxicity, including 37\% achieving CR or PR. There was no correlation between treatment response and baseline driver muts or DIPSS. Of 8 poor responders, 3 had ASXL1 or SRSF2. 


\section{Conclusion:}

Early treatment with rIFNa of pts without HMR may prevent developing marked splenomegaly, anemia, and florid myelofibrosis. Molecular profile at diagnosis may predict prognosis and treatment response.

Keywords: Early primary and secondary myelofibrosis, interferon (rIFNa) treatment, splenomegaly, marrow morphology, molecular profile, high molecular risk

\section{Introduction:}

The successful treatment of early MF (eMF) with rIFNa has been reported by us and others, but in these studies, the effect of driver (i.e. JAK2, CALR and MPL) and epigenetic or other muts at diagnosis were not considered and systemic marrow examinations were not performed. ${ }^{1-4}$. The molecular abnormalities of pts with myelofibrosis have recently been emphasized, and their incorporation into a new prognostic scoring system has been recommended as an improvement over the DIPSS (Dynamic International Prognostic Scoring System) or its variations. ${ }^{5,6}$. We now report 30 pts, expanded from our initial cohort of 17 , whose molecular abnormalities at baseline were correlated with hematologic and marrow response and survival following rIFNa treatment.

Molecular abnormalities at diagnosis have been investigated in pts with both primary (PMF) and secondary myelofibrosis (SMF)..$^{5,6,7-11}$. No correlation between initial 
mutation status and response to treatment with ruxolitinib or overall survival was reported in pts with PMF or SMF. ${ }^{7,8}$ Patel et. al. showed that pts with three or more muts had a lower overall survival and a shorter time to treatment discontinuation. ${ }^{7}$ Furthermore, while individual driver muts, had no correlation with response to treatment with ruxolitinib, spleen response ( $\geq 50 \%$ reduction in palpable splenomegaly) was inversely correlated with the number of muts derived from a standard myeloid panel. Furthermore, patients with one or more muts in ASXL1, EZH2, and IDH1/2 were significantly less likely to have a spleen response than those with none. ${ }^{7}$

Recently, a number of muts have been identified categorizing pts as "high molecular risk" (HMR). ${ }^{5}$ Specifically, these muts include ASXL1, EZH2, SRSF2, and IDH1/2 which have been associated with both shorter overall survival and increased risk of transformation to acute leukemia. ${ }^{5}$ Further, two or more muts were associated with a worse prognosis than one or none. ${ }^{9}$ Although these secondary epigenetic abnormalities have been generally accepted as associated with an adverse prognosis, their association with response to treatment with rIFNa has never been examined.

Patients with PMF and CALR muts reportedly have a better overall survival than those with JAK2, MPL, or no driver muts, whereas triple negative pts have a significantly shorter survival. ${ }^{6,10}$ In multivariate analysis including both driver and epigenetic abnormalities, only CALR and ASXL1 muts remained prognostically significant; CALR had a favorable impact on prognosis, and ASXL1 a negative one. ${ }^{9}$. It was therefore proposed that $\operatorname{CALR}(-) \operatorname{ASXL1(+)}$ and triple negative PMF be considered HMR. These pts, however, were not treated uniformly. 
We now report our results of our single-center study of 30 pts with either primary or secondary MF correlating the initial molecular and epigenetic profile with clinical and hematologic response to rIFNa.

\section{Material and Methods:}

The study was approved by our Institutional Review Board; it was performed according to the Declaration of Helsinki. Pts were diagnosed using World Health Organization (WHO) criteria for PM and IWG-MRT criteria for post polycythemia vera and post essential thrombocythemia myelofibrosis (post-PV MF, post-ET MF). ${ }^{12,13}$ Evaluation included medical history, physical examination, complete blood and differential counts, routine serum chemistries, liver, renal, and thyroid function tests, bone marrow biopsy, BCR-ABL determination, cytogenetic evaluation, epigenetic testing and JAK2, CALR, and MPL analysis. Spleen size was measured in centimeters below the left costal margin (LCM) in the midclavicular line.

Prognosis was assessed using the IWG-MRT's Dynamic International Prognostic Scoring System (DIPSS). ${ }^{14}$ We used DIPSS instead of the more recent DIPSS-PLUS, ${ }^{15}$ since by definition, none of our early phase pts required any transfusions at study onset. Treatment response was assessed using the consensus criteria of the IWG-MRT and European LeukemiaNet (ELN). ${ }^{16}$. Response classifications included: complete remission $(\mathrm{CR})$, partial remission (PR), clinical improvement (Cl), stable disease (SD), and progressive disease (PD). 
Marrow specimens were stained with hematoxylin-eosin, Giemsa, and with silver and trichrome for reticulin, and collagen. Fibrosis was graded using the European Consensus System. ${ }^{17}$. Criteria for inclusion in this study required either low or intermediate 1 prognostic score and residual hematopoietic foci occupying $\geq 15 \%$ of the marrow biopsy. Marrow aspirate was submitted for cytogenetic analysis when sufficient material could be obtained.

JAK2 and CALR muts were detected and/or quantified by pyrosequencing and MiSeq analysis, respectively, according to described methods. ${ }^{18-20}$ JAK2(-) pts were retrospectively evaluated for CALR and MPL muts.

Genomic DNA extracted from frozen mononuclear cells isolated from peripheral blood collected at the time of diagnosis. Next-generation sequencing was performed using a custom 45-gene myeloid malignancy targeted amplicon enrichment panel (RainDance Technologies, Billerica, MA) inclusive of 45 genes recurrently mutated in myeloid malignancies (ABL1, ASXL1, BCOR, BRAF, CALR, CBL, CEBPA, DNMT3A, ETV6, EZH2, FAM5C, FLT3, GATA1, GATA2, HNRNPK, IDH1, IDH2, IKZF1, JAK1, JAK2, KDM6A, KIT, KRAS, MPL, NFE2, NOTCH1, NPM1, NRAS, PHF6, PTPN11, RAD21, RUNX1, SETBP1, SF3B1, SH2B3, SMC1A, SMC3, SRSF2, STAG2, SUZ12, TET2, TP53, U2AF1, WT1, ZRSR2). Amplicons were sequenced on an Illumina MiSeq (v3 chemistry) using 260 bp paired-end reads followed by alignment to the human reference genome (hs37d5) using BWA MEM. ${ }^{21}$. Mutations were identified using VarDict ${ }^{22}$ excluding primer regions and annotated using SnpEff $4.1 .{ }^{23}$. Common SNPS likely to be non-somatic with minor allele frequency greater than $0.25 \%$ were excluded from subsequent analysis with the exception of variants identified at least twice in the 
Catalog of Somatic Mutations in Cancer (COSMIC) v68. ${ }^{24}$ The cutoff point for mutation positive status for all muts was $5 \%$.

Descriptive statistics (including mean, standard deviation, median, range, frequency, and percent) were calculated to characterize the study cohort. The relationship between various definitions of mut status and 1$)$ response (CR/PR/CI/SD vs. relapse/PD/death; $\mathrm{CR} / \mathrm{PR} / \mathrm{Cl}$ vs. SD/PD/relapse/death) and 2) prognostic factors of interest were assessed by Fisher's exact test. Kaplan-Meier survival analysis was performed to assess overall survival (OS) and progression-free survival (PFS). All pvalues are two-sided with statistical significance evaluated at the 0.05 alpha level. All analyses were performed in SPSS Version 24.0 (SPSS Inc., Chicago, IL) and Stata Version 14.0 (StataCorp, College Station, TX).

Either rIFNa-2b (Intron®) or pegylated IFNa-2a (Pegasys ${ }^{\circledR}$ ) was employed depending upon insurance coverage and pt desire. Eighteen pts initially received rIFNa2b 500,000 to 1 million units (mu) subcutaneously 3 times weekly (TIW), gradually increasing the dose to 2 to 3 mu TIW as tolerated and to reduce spleen size, which was used as a clinical indicator of response. Seven pts received 45 or $90 \mu \mathrm{g}$ of peg-IFNa-2a weekly. Five pts began treatment with rIFNa-2b, but treatment was changed to pegIFNa-2a (at the aforementioned doses) after a median of two years because of their preference and insurance availability. Contraindications to initiating rIFNa included severe depression, neuropathy, active thyroid dysfunction, current or past history of autoimmune disease, current or past history of inflammation-mediated comorbidities, and significant hepatic, renal, or cardiac abnormalities. Adjustment and final selection of a maintenance dose was based on clinical and marrow response, tolerability, and 
toxicity which was assessed using the Common Terminology Criteria, Version $4.0(\mathrm{NCl}$, NIH, DHHS. May 29, 2009).

\section{Results:}

Of 96 pts meeting the 2008 WHO criteria for PMF or IWG-MRT criteria for SMF, 66 were excluded owing to marked marrow fibrosis and $<15 \%$ hematopoietic foci or unwillingness to be treated with interferon in the early phase of their disease. ${ }^{11,12}$. The remaining 30 pts (31\%), met our study inclusion criteria. There were 16 women and 14 men, whose median age at the time of diagnosis was 58 years (range, 34-72 years) (Table I). Twenty-one pts (70\%) met the WHO criteria for PM, 7 (23\%) for post-PV MF, and $2(7 \%)$ for post-ET MF. ${ }^{11,12}$. There were $22 \mathrm{JAK}^{\mathrm{V} 617 \mathrm{~F}}(+), 6 \mathrm{CALR}(+)$, and $2 \mathrm{MPL}(+)$ pts. Of the CALR(+) pts, 5 had the 52 bp (Type 1) deletion and one a 19 bp deletion, and of the MPL(+) pts, one had a W515K variant, and the other, W515L. All 7 post-PV MF pts were JAK2 ${ }^{\mathrm{V} 617 F}(+)$. Of post-ET MF pts, 1 was JAK2 ${ }^{\operatorname{V617F}}(+)$ and 1 CALR(+). Of the PM pts, 14 were $\mathrm{JAK}^{\mathrm{V} 617 \mathrm{~F}}(+), 5 \operatorname{CALR}(+)$, and $2 \mathrm{MPL}(+)$.

Twenty-two pts were classified as low-risk, and 8 as intermediate 1 risk. Median baseline leukocyte, hematocrit, hemoglobin, and platelet values were $8.3 \times 1000 / \mu \mathrm{L}$, $38.4 \%, 12.9 \mathrm{~g} / \mathrm{dL}$, and $345,500 / \mu \mathrm{L}$, respectively. Median values at last follow-up were $6.7 \times 1000 / \mu \mathrm{L}, 36 \%, 12 \mathrm{~g} / \mathrm{dL}$, and $279,000 / \mu \mathrm{L}$. Median duration of disease prior to rIFNa treatment was 1.9 years since PMF or SMF diagnosis, and median duration of treatment to date has been 5.6 years.

Of 30 pts, 2 (7\%) achieved CR, 9 (30\%) PR, 4 (13\%) Cl and 7 (23\%) SD (Tables II and III). Four (13\%) pts had progressive disease and 4 (13\%) died: 3 of progressive 
disease (PD), although one was not compliant with respect to rIFNa use, and one died 6 weeks post bone marrow transplant. Nine of 22 low risk pts achieved CR or PR, while only 2 of 8 intermediate 1 did.

Baseline and follow-up spleen measurements were available in all pts. Of 30 pts, $8(27 \%)$ had no splenomegaly at onset of rIFNa therapy; 6 of these $8(75 \%)$ pts remained without splenomegaly during their treatment (median, 5.5 years). Six of 10 pts (60\%) with "slight" splenomegaly ( $<4 \mathrm{~cm}$ BLCM), achieved non-palpable spleens after a median of 10.8 years of therapy. Both pts with moderate splenomegaly (spleen size: 4 $9 \mathrm{~cm}$ BLCM) showed an increase in spleen size (median 4.6 years). Of the 10 pts with marked splenomegaly (> $9 \mathrm{~cm}$ BLCM), 4 (40\%) responded with at least a $50 \%$ reduction in spleen size (median duration of treatment: 5.7 years). The remaining 6 pts (60\%) had either a slight decrease or stable spleen size. Overall, the 18 pts with a spleen size less than $4 \mathrm{~cm}(60 \%)$ had a better response to treatment than pts with significant splenomegaly; 2 achieved CR, $9 \mathrm{PR}$, and $1 \mathrm{Cl}, 4$ remained stable, 1 progressed and 1 died. Of the non-responders, 2 pts had a HMR, one of which had 3 muts. On the other hand, of the 12 pts with significant or moderate baseline splenomegaly, including 2 with a HMR and 3 muts and 1 pt with 4 muts 3 achieved $\mathrm{Cl}, 3$ remained stable, 3 progressed, and 3 died. In summary, a large spleen prior to the start of therapy was associated with worse progression-free survival and poorer response to rIFNa therapy $(p=0.05$, log-rank test), however, pts with a small spleen and HMR did not respond to rIFNa.

Toxicity was generally mild, grade 1 or 2 , dose-related, and subsided or diminished upon dose reduction (Table IV). These included mild depression, dry skin, 
cough, myalgia, fatigue and asthenia. Overall, the myelosuppressive effects of rlFNa were mild. Seventeen pts developed increasing anemia (13: grade 1; 4: grade 2); one had grade 3 thrombocytopenia. In these cases, doses of interferon were temporarily reduced or interrupted until cytopenias resolved. One pt developed hyperthyroidism and treatment was discontinued.

Follow-up BMBs were possible in 25 of 30 pts (83\%). Reduction in cellularity occurred in 12, an increase in 3 , and no change in 8 (baseline cellularity unreported for 2). Five pts had a reduction in reticulin fibrosis, 10 remained stable, and 9 pts had an increase (baseline fibrosis score was unreported for one). Unequivocal improvement in marrow morphology occurred in 12 of $30(40 \%)$. However, even in the 2 patients categorized as CR according to ELN criteria, subtle abnormalities in megakaryocyte nuclear structure persisted. Of 11 pts who had reduction in splenomegaly and also evaluable BMBs, 8 had reduction in cellularity (73\%), 2 showed no change, and one increased in cellularity. In general, therefore, the majority of pts with a reduction in spleen size had an improvement in marrow morphology.

Of the 30 pts, baseline marrow cytogenetic analyses were available in 22, and sequential cytogenetic analyses in 15 . Of 13 pts with normal cytogenetics at baseline, one developed $-13 q$, one trisomy 9 and the rest remained normal. Two pts had baseline cytogenetic abnormalities that did not change over the course of treatment: one had trisomy 9 , and one $-13 q$. These cytogenetic abnormalities have been reported previously to be favorable in this disease..$^{25-27}$

No statistically significant differences occurred between the type of driver mut and response to treatment (IWG-MRT) (Fisher exact test, p-value $=0.65)$. Of the $30 \mathrm{pts}$, 
$22(73 \%)$ were $\mathrm{JAK} 2^{\mathrm{V} 617 \mathrm{~F}}(+)$. There was no significant correlation between driver mut and DIPSS score at baseline (data not shown).

The mean JAK2 mutant allele burden at baseline for the low and intermediate-1 risk groups were $47.6 \%$ (range: $2-93 \%$ ) and $48.1 \%$ (10.1-95.3\%), respectively. The range of baseline mutant allele burden was also similar in both groups. Of 18 follow-up specimens, 5 showed both a median mutant allele burden decrease of $23 \%$ (range, 9$58 \%)$ and a decrease in marrow cellularity after a median treatment duration of $7 \mathrm{yrs}$ (range: $1.5-12$ yrs). There was no consistent or statistical correlation between decrease in JAK2 ${ }^{\mathrm{V} 617 \mathrm{~F}}$ allele burden, clinical response, marrow fibrosis, or spleen size (data not shown).

Additional mut analysis was possible in 25 of 30 pts (83\%) (Table III). Of 12 pts with $\mathrm{Cl}$ or better, none had a known HMR mut. Of 6 pts who remained stable, 1 had a HMR mut (ASXL1). Of 8 pts who progressed or died, 7 were tested by the NGS panel and 3 had HMR (ASXL1 and SRSF2). Pts with known HMR had worse responses than those without; of 4 pts with such muts, 1 progressed and 2 died. The other remained stable. All 4 of these pts had PM. Pts with 3 or more muts did worse than those with fewer. Of 5 pts with 3 or more muts, 2 progressed and 2 died; all 4 had PMF. The other, who had post-PV MF, had PR. In addition, pts without the ASXL1mut seemed to do better than pts with it, although sample size precludes a definitive statement $(p=0.19)$.

In this selected group of pts, OS and PFS at 5 years were $91.5 \%$ and $88 \%$, respectively (Figure 1).

\section{Discussion:}


This is the first study correlating initial driver and epigenetic muts with documented clinical and marrow responses to rIFNa therapy. Our results suggest that therapeutic intervention with rIFNa prior to the development of clinically significant adverse features such as marked splenomegaly, anemia, and florid myelofibrosis and osteosclerosis is beneficial in pts with early stage disease. The majority of these pts improved or remained stable (73\%), regardless of whether the initial DIPSS score was low or intermediate 1, suggesting that treatment with low-dose rIFNa should be initiated prior to the development of the cascading events preceding features of irreversible disease. In this selected group of pts, OS and PFS were impressive but of course, inconclusive.

Initial driver muts did not correlate with response to treatment, consistent with the response of ruxolitinib-treated MF pts; no correlation between type of genetic mut and spleen response or survival was noted in such pts, however there was an inverse correlation between number of initial muts and response. ${ }^{7}$ CALR $(+)$ pts did not have superior response or survival, differing from the findings of others who observed a better overall survival and lower risk of thrombosis in $\operatorname{CALR}(+)$ pts compared with JAK2 (+), $\operatorname{MPL}(+)$, or triple negative pts. ${ }^{2,7}$ This study neither included a full myeloid mut evaluation nor treatment specification. This is important because the lack of correlation between mutational status and response to treatment may be therapy-specific and not a generality, possibly accounting for the varying results to date correlating driver and epigenetic muts and clinical results in MF. It is of interest, however, that our longest surviving patient, in CR more than $25 \mathrm{yrs}$, has a molecular profile including CALR(+) and TET2(+). 
Thus, although baseline driver mut status was not useful prognostically, a molecular profile at diagnosis should be performed to identify HMR abnormalities since these negatively affected response to treatment, including rIFNa. Three of 4 pts with HMR abnormalities (ASXL1 or SRSF2) had a poor response, all with PM, compared with only 4 poor responders in 20 LMR pts.

The use of low-dose rIFNa in "early" PM, post-ET MF and post-PV MF, as defined, resulted in marrow reversion, regression of splenomegaly, and disease stabilization, with tolerable toxicity in $73 \%$ of pts. Reduction in splenomegaly appears predictive of improvement in marrow morphology and may be used as a guide for continuing therapy in the absence of HMR. We emphasize that long-term treatment must be anticipated when using low-dose rIFNa. Median duration of therapy was 5.6 years and is still ongoing for approximately $50 \%$ of the pts. That subtle but definite structural megakaryocytic abnormalities persist in pts who are considered CR suggests that "biologic remission" but not "cure" has been achieved, analogous to the responses in $\mathrm{CML}$ and $\mathrm{PV} .{ }^{28}$. It is therefore perhaps more appropriate to call this a complete response, as opposed to remission.

The OS of PM pts treated with rIFNa was favorable compared with those treated with a variety of therapies. ${ }^{29}$. In a group of 208 low and intermediate 1 risk pts, median survival was 10.6 yrs, whereas median survival for our pts was not yet reached and OS at $10.6 \mathrm{yrs}$ is $72.7 \%$. This suggests that early treatment with rIFNa is not harmful, and may offer survival benefit, despite the usual caveats of analyzing survival in a small cohort with relatively few deaths. 
Although it has been argued that "observation only" is most appropriate for lowrisk pts because of their long survival, such a recommendation does not account for interval symptoms and morbidity during the course of the disease. ${ }^{30}$. We acknowledge that in following our treatment recommendations, a measurable cohort of pts may receive rIFNa prematurely. On the other hand, a significant number of pts who will require the drug may not. Unhappily, this is true for many other forms of chemotherapy. The symptom burden in pts with MF has long been emphasized and must be considered for all pts. ${ }^{31}$ Unquestionably, the future requires much-improved risk adaptive therapy, hopefully on a molecular basis. Although our results are encouraging, patient selection and sample size play a critical role, requiring confirmation in a larger and preferably randomized trial. However, the importance and value of single-arm studies in orphan diseases has recently been analyzed and discussed. ${ }^{32,33}$. However, until further systematic evaluation of IFN in early myelofibrosis, it remains an experimental therapy. 
Table I. Baseline data

\begin{tabular}{|c|c|c|c|c|}
\hline Characteristic & $\begin{array}{l}\text { All patients } \\
\qquad \mathrm{N}=30\end{array}$ & $\underset{\mathrm{N}=22}{\mathrm{JAK}^{\mathrm{V} 617 \mathrm{~F}}}(+)$ & $\begin{array}{c}\operatorname{CALR}(+) \\
\mathrm{N}=6\end{array}$ & $\begin{array}{c}\mathrm{MPL}(+) \\
\mathrm{N}=2\end{array}$ \\
\hline Age at diagnosis (yrs), median (range) & $58(34-73)$ & $58(34-72)$ & $55(35-60)$ & $62(61-62)$ \\
\hline Sex (female/male) & $16 / 14$ & $13 / 9$ & $4 / 2$ & $0 / 2$ \\
\hline \multicolumn{5}{|l|}{ Baseline characteristics } \\
\hline $\mathrm{Hb}(\mathrm{g} / \mathrm{dL})$, median (range) & $12.9(8.7-15.6)$ & $13(8.7-15.6)$ & $10.9(10.4-13.2)$ & $13.4 / 14.2$ \\
\hline $\operatorname{WBC}\left(10^{3} / L\right)$, median (range) & $9(2.8-36.8)$ & $10.8(5-36.8)$ & $7.1(3.8-13.2)$ & $5.9 / 11$ \\
\hline Plt $\left(10^{3} / L\right)$, median (range) & $345(111-1155)$ & $322(123-1155)$ & $445(111-570)$ & $156 / 226$ \\
\hline Hct (\%), median (range) & $38.4(26.8-51.7)$ & $39(26.8-51.7)$ & $33.3(32.1-38.7)$ & $39.3 / 41.8$ \\
\hline PBBL (\%), median (range) & $0(0-10)$ & $0(0-10)$ & $0(0-2)$ & $0 / 1$ \\
\hline Spleen (cm), median (range) & $2(0-32)$ & $3(0-32)$ & $1.5(0-8)$ & $0 / 16$ \\
\hline JAK2 Allele Burden (\%), median (range) & --- & $46.2(4.9-95.3)$ & --- & --- \\
\hline DIPSS Low & 22 & 15 & 5 & 2 \\
\hline DIPSS Int-1 & 8 & 7 & 1 & 0 \\
\hline \multicolumn{5}{|l|}{ Follow-up characteristics* } \\
\hline Therapy duration (months), median (range) & $80.3(10-247)$ & $70(19-156)$ & $75(41-247)$ & $49 / 64$ \\
\hline $\mathrm{Hb}(\mathrm{g} / \mathrm{dL})$, median (range) & $11.8(6.1-14.7)$ & $11.8(6.1-14.7)$ & $12(10.1-13.5)$ & $9.8 / 12.8$ \\
\hline WBC $\left(10^{3} / L\right)$, median (range) & $7.0(2.2-40.7)$ & $6.3(2.5-40.7)$ & $7.1(4.1-40.7)$ & $2.2 / 8.1$ \\
\hline PIt $\left(10^{3} / L\right)$, median (range) & $279(41-865)$ & $279(66-599)$ & $488(41-865)$ & $42 / 154$ \\
\hline Hct (\%), median (range) & $36.4(18.8-45.2)$ & $35.8(18.8-45.2)$ & $36.6(30.2-39.6)$ & $29.1 / 37.6$ \\
\hline PBBL (\%), median (range) & $0(0-6)$ & $0(0-6)$ & $0(0-2)$ & $0 / 2$ \\
\hline Spleen (cm), median (range) & $2(0-32)$ & $2(0-32)$ & $0(0-14)$ & $0 / 16$ \\
\hline JAK2 Allele Burden (\%), median (range) & --- & $42.4(4.1-94.5)$ & --- & --- \\
\hline
\end{tabular}

*Either at the end of treatment or date of last follow-up for ongoing patients.

PBBL: peripheral blood blast count

Spleen measured in $\mathrm{cm}$ below the left costal margin. 
Table II. IWG-MRT treatment response categorized by DIPSS at baseline

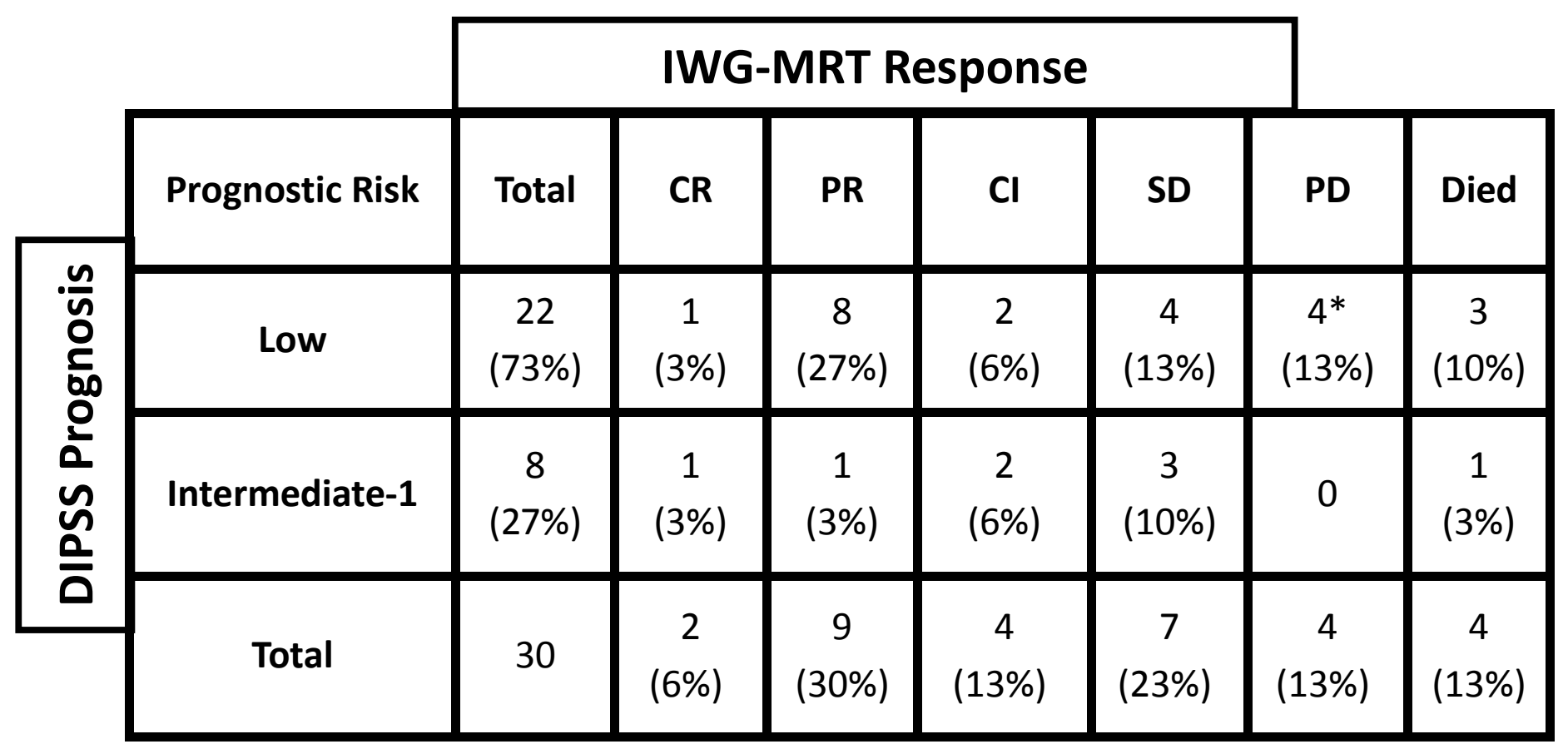

$\mathrm{CR}=$ Complete response

$\mathrm{PR}=$ Partial response

$\mathrm{Cl}=$ Clinical improvement

$\mathrm{SD}=$ Stable disease

$\mathrm{PD}=$ Progressive disease

* 3 PD, 1 relapse (splenomegaly)

Overall, 22/30 (73\%) had a response or remained stable

IWG-MRT consensus criteria for treatment response in myelofibrosis with myeloid metaplasia. Tefferi, et al. Blood 2006. 
Table III. Response to treatment categorized by baseline driver, epigenetic and other mutations

\begin{tabular}{|c|c|c|c|c|c|c|c|c|}
\hline & & Total & CR & PR & $\mathrm{Cl}$ & SD & PD & Death \\
\hline \multirow[t]{9}{*}{ JAK2 } & Single mut & 5 & 1 & 2 & & & 1 & 1 \\
\hline & +ASXL1 & 1 & & & & 1 & & \\
\hline & $+\mathrm{ASXL1+MISC}$ & 1 & & & & & 1 & \\
\hline & +DNMT3A & 2 & & & 1 & 1 & & \\
\hline & $+\mathrm{MISC}$ & 1 & & & 1 & & & \\
\hline & $+\mathrm{SH} 2 \mathrm{~B} 3$ & 1 & & & & 1 & & \\
\hline & + TET2 & 4 & & & 1 & 2 & 1 & \\
\hline & +TET2+MISC & 1 & & 1 & & & & \\
\hline & $+\mathrm{WT} 1$ & 1 & & & & 1 & & \\
\hline \multirow[t]{5}{*}{ CALR } & Single mut & 2 & & 2 & & & & \\
\hline & +ASXL1+SETBP1 & 1 & & & & & & 1 \\
\hline & $+\mathrm{MISC}$ & 1 & & 1 & & & & \\
\hline & +SETBP1+KDM6A+FLT3 & 1 & & & & & 1 & \\
\hline & + TET2 & 1 & 1 & & & & & \\
\hline \multirow[t]{3}{*}{ MPL } & Single mut & 1 & & 1 & & & & \\
\hline & +SRSF2+TET2 & 1 & & & & & & 1 \\
\hline & Total & 25 & 2 & 7 & 3 & 6 & 4 & 3 \\
\hline
\end{tabular}

$\mathrm{CR}=$ Complete response

$\mathrm{PR}=$ Partial response

$\mathrm{Cl}=$ Clinical improvement

$\mathrm{SD}=$ Stable disease

PD $=$ Progressive disease

IWG-MRT consensus criteria for treatment response in myelofibrosis with myeloid metaplasia. Tefferi, et al. Blood 2006. 
Table IV. Side-effects of rIFNa treatment

\begin{tabular}{|c|c|c|c|}
\hline Toxicity Type & Symptoms Experienced & \# Pts. * & Grade \\
\hline \multirow[t]{7}{*}{ Systemic } & & & $1-2$ \\
\hline & Aesthenia, Fatigue, Myalgia, Weakness & 18 & $\begin{array}{l}\text { 1-3 (17 pts.: grade } \\
1-2)\end{array}$ \\
\hline & Alopecia, Dry Skin, Itching & 6 & $1-2$ \\
\hline & Cough, Dyspnea & 3 & $1-2$ \\
\hline & Nausea & 4 & 1 \\
\hline & Palpitations, Dizziness & 6 & $1-2$ \\
\hline & Depression, Neuropathy & 9 & $1-2$ \\
\hline \multirow[t]{4}{*}{ Hematologic } & & & 1-4 \\
\hline & Anemia & 17 & $1-2$ \\
\hline & Thrombocytopenia & 12 & $\begin{array}{l}1-4\{10 \text { pts.: grade } \\
1-2)\end{array}$ \\
\hline & Leukopenia & 9 & $1-2$ \\
\hline \multirow[t]{6}{*}{ Metabolic } & & & $1-2$ \\
\hline & Serum liver function abnormalities: & & \\
\hline & $\uparrow$ Aspartate aminotransferase (AST) & 10 & 1 \\
\hline & $\uparrow$ Alkaline Phosphatase & 7 & 1 \\
\hline & $\uparrow$ Alanine transaminase (ALT) & 7 & $1-2$ \\
\hline & Hyperthyroidism & 1 & \\
\hline
\end{tabular}




\section{References}

1. Silver R, Vandris K, Goldman J. Recombinant interferon-a may retard progression of early primary myelofibrosis: a preliminary report. Blood. 2011;117(24):6669-6672.

2. Kiladjian J, Mesa R, Hoffman R. The renaissance of interferon therapy for the treatment of myeloid malignancies. Blood. 2011;117(18)4706-4715.

3. Gowin K, Thapaliya P, Samuelson J, et. al. Experience with pegylated interferon $\mathrm{a}-2 \mathrm{a}$ in advanced myeloproliferative neoplasms in an international cohort of 118 patients. Haematologica. 2012;97(10):1570-1573.

4. Ianotto JC, Kiladjian JJ, Demory JL, et. al. PEG-IFN-alpha-2a therapy in patients with myelofibrosis: a study of the French Groupe d'Etudes des Myelofibroses (GEM) and France Intergroupe des syndromes Myéloprolifératifs (FIM). Br J Haematol. 2009;146(2):223-225.

5. Vannucchi AM, Lasho TL, Guglielmelli P, et. al. Mutations and prognosis in primary myelofibrosis. Leukemia. 2013;27(9):1861-1869.

6. Rumi E, Pietra D, Pascutto C, et. al. Clinical effect of driver mutations of JAK2, CALR, or MPL in primary myelofibrosis. Blood. 2014;124(7):1062-1069.

7. Patel KP, Newberry KJ, Luthra R, et. al. Correlation of mutation profile and response in patients with myelofibrosis treated with ruxolitinib. Blood. Prepublished June 29, 2015, as DOI 10.1182/blood-2015-03-633404. 
8. Guglielmelli P, Biamonte F, Rotunno G, et. al. Impact of mutational status on outcomes in myelofibrosis patients treated with ruxolitinib in the COMFORT-II study. Blood. 2014;123(14): 2157-2160

9. Guglielmelli P, Lasho TL, Rotunno G, et. al. The number of prognostically detrimental mutations and prognosis in primary myelofibrosis: an international study of 797 patients. Leukemia. 2014;28(9):1804-1810.

10. Tefferi A, Guglielmelli P, Lasho TL, et. al. CALR and ASXL1 mutations-based molecular prognostication in primary myelofibrosis: an international study of 570 patients. Leukemia. 2014;28(7):1494-1500.

11. Rotunno G, Pacilli A, Artusi V, et. al. Epidemiology and clinical relevance of mutations in postpolycythemia vera and postessential thrombocythemia myelofibrosis: A study on 359 patients of the AGIMM group. Am J Hematol. 2016;91(7)681-686.

12. Tefferi A, Thiele J, Orazi A, et. al. Proposals and rationale for revision of the World Health Organization diagnostic criteria for polycythemia vera, essential thrombocythemia, and primary myelofibrosis: recommendations from an ad hoc international expert panel. Blood. 2007;110(4):1092-1097.

13. Barosi G, Mesa RA, Thiele J, et. al. Proposed criteria for the diagnosis of postpolycythemia vera and post-essential thrombocythemia myelofibrosis: a consensus statement from the International Working Group for Myelofibrosis Research and Treatment. Leukemia. 2008;22(2):437-438. 
14. Passamonti F, Cervantes F, Vannucchi AM, et. al. A dynamic prognostic model to predict survival in primary myelofibrosis: a study by the IWG-MRT (International Working Group for Myeloproliferative Neoplasms Research and Treatment). Blood. 2010; 115(9);1703-1708.

15. Gangat N, Caramazza D, Vadya R, et. al. DIPSS plus: a refined Dynamic International Prognostic Scoring System for primary myelofibrosis that incorporates prognostic information from karyotype, platelet count, and transfusion status. J Clin Oncol. 2011;29(4):392-397.

16. Tefferi A, Cervantes F, Mesa R, et. al. Revised response criteria for myelofibrosis: International Working Group-Myeloproliferative Neoplasms Research and Treatment (IWG-MRT) and European LeukemiaNet (ELN) consensus report. Blood. 2013;122(8), 1395-1398.

17. Thiele J, Kvasnicka HM, Facchetti F, Franco V, van der Walt J, Orazi A. European Consensus on grading bone marrow fibrosis and assessment of cellularity. Haematologica. 2005;90(8):1128-1132.

18. Chen Q, Lu P, Jones AV, Cross NC, Silver RT, Wang YL. Amplification refractory mutation system, a highly sensitive and simple polymerase chain reaction assay, for the detection of JAK2V617F mutation in chronic myeloproliferative disorders. J Mol Diagn. 2007;9(2):272-276.

19. Jones AV, Silver RT, Waghorn K, et. al. Minimal molecular response in polycythemia vera patients treated with imatinib or interferon alpha. Blood. 2006;107(8):3339-3341. 
20. Jones AV, Ward D, Lyon M, et. al. Evaluation of methods to detect CALR mutations in myeloproliferative neoplasms. Leuk Res. 2015;39(1):82-87.

21. Li, H. Aligning sequence reads, clone sequences and assembly contigs with BWA-MEM. arXiv Preprint arXiv, 00, 3. http://doi.org/arXiv:1303.3997 [q-bio.GN]

22. Lai Z, Markovets A, Ahdesmaki M, et. al. VarDict: a novel and versatile variant caller for next-generation sequencing in cancer research. Nucleic Acids Res. 2016;44(11)e108

23. De Baets G, Van Durme J, Reumers J, et. al. SNPeffect 4.0: On-line prediction of molecular and structural effects of protein-coding variants. Nucleic Acids Res. 2012; 40(Database issue):D935-D939.

24. Forbes SA, Beare D, Gunasekaran P, et. al. COSMIC: Exploring the world's knowledge of somatic mutations in human cancer. Nucleic Acids Res, 2015;43(Database issue):D805-D811.

25. Tefferi A, Mesa R, Schroeder G, et. al. Cytogenetic findings and their clinical relevance in myelofibrosis with myeloid metaplasia. $\mathrm{Br} \mathrm{J}$ Haematol. 2001;113(3):763-771.

26. Tefferi A, Dingli D. Li C-Y, Dewald GW. Prognostic Diversity among Cytogenetic Abnormalities in Myelofibrosis with Myeloid Metaplasia. Cancer. 2005;104(8_1656-1660. 
27. Tam CS, Abruzzo LV, Lin KI, et. al. The role of cytogenetic abnormalities as a prognostic marker in primary myelofibrosis: applicability at the time of diagnosis and later during disease course. Blood. 2009;113(18):4171-4178.

28. Pizzi M, Silver RT, Barel A, Orazi A. Recombinant interferon- $\alpha$ in myelofibrosis reduces bone marrow fibrosis, improves its morphology and is associated with clinical response. Mod Pathol. 2015;28(10):1315-1323.

29. Cervantes F, Dupriez B, Passamonti F. Improving survival trends in primary myelofibrosis: an international study. J Clin Oncol. 2012;30(24): 2981 - 2987.

30. Tefferi A. Primary myelofibrosis: 2014 update on diagnosis, risk-stratification, and management. Am J Hematol. 2014;89(9):915-925.

31. Mesa RA, Niblack J, Wadleigh M, et. al. The burden of fatigue and quality of life in myeloproliferative disorders (MPDs). Cancer. 2007;109(1):68-76.

32. Hasselbalch HC, Silver RT. Interferon in polycythemia vera and related neoplasms. Can it become the treatment of choice without a randomized trial? Expert Rev Hematol. 2015;8(4):439-445.

33. Silver RT, Hasselbalch HC. Optimal therapy for polycythemia vera and essential thrombocythemia: Preferred use of interferon therapy based on phase 2 trials. Hematology. 2016;21(7):387-391. 